\title{
Unsupervised Anomaly Detection on Node Attributed Networks: A Deep Learning Approach
}

\author{
Parsa Kavehzadeh ${ }^{*}$ \\ Computer Engineering Department, \\ Amirkabir University of Technology, \\ Iran
}

\author{
Mohammadreza Samadi* \\ Computer Engineering Department, \\ Amirkabir University of Technology, \\ Iran
}

\author{
Maryam Amir Haeri \\ Learning, Data-Analytics and \\ Technology Department, University \\ of Twente, Netherlands
}

\begin{abstract}
Anomaly detection has been one of the important issues in social network analysis in recent years due to the crucial role it plays in different applications such as fraud and spammer detection. Using both graph and node characteristics leads to more accurate results in detecting anomalous nodes of node attributed networks. Most of the research works in this field are concentrated on supervised methods for anomaly detection. However, in real-world problems, there is not enough labeled data to use supervised methods for anomaly detection. This paper proposes an unsupervised method for detecting anomalous nodes in node attributed networks. The methods used a two-step deep learning approach. In the first step, structural features of the network are extracted using node2vec; in the next step, Variational AutoEncoder (VAE) is used to detect the anomalies considering both structural and node attributes. The anomalous nodes are recognized by their higher reconstruction loss. Our experimental results on two datasets, BlogCatalog and Flickr, show that the suggested method can compete with the state-of-theart approaches of anomaly detection in attributed networks. Our method (Deep ${ }^{2} \mathrm{NAD}$ ) outperforms the state-of-the-art result on the Flickr dataset based on AUC. Moreover, it receives an acceptable AUC over the BlogCatalog dataset in comparison to the state-ofthe-art methods.
\end{abstract}

\section{CCS CONCEPTS}

- Computing methodologies; • Machine learning; • Learning paradigms; • Unsupervised learning; • Anomaly detection;

\section{KEYWORDS}

Attributed Networks, Anomaly Detection, Unsupervised Learning, Deep Learning

\section{ACM Reference Format:}

Parsa Kavehzadeh, Mohammadreza Samadi, and Maryam Amir Haeri. 2021. Unsupervised Anomaly Detection on Node Attributed Networks: A Deep Learning Approach. In 2021 The 4th International Conference on Information

\footnotetext{
${ }^{*}$ The first two authors contributed equally to this research.

Permission to make digital or hard copies of all or part of this work for personal or classroom use is granted without fee provided that copies are not made or distributed for profit or commercial advantage and that copies bear this notice and the full citation on the first page. Copyrights for components of this work owned by others than ACM must be honored. Abstracting with credit is permitted. To copy otherwise, or republish, to post on servers or to redistribute to lists, requires prior specific permission and/or a fee. Request permissions from permissions@acm.org.

ICISS 2021, March 17-19, 2021, Edinburgh, United Kingdom

(c) 2021 Association for Computing Machinery.

ACM ISBN 978-1-4503-8913-6/21/03..\$15.00

https://doi.org/10.1145/3459955.3460597
}

Science and Systems (ICISS 2021), March 17-19, 2021, Edinburgh, United Kingdom. ACM, New York, NY, USA, 6 pages. https://doi.org/10.1145/3459955. 3460597

\section{INTRODUCTION}

With the emergence of online intertwined networks, detecting anomalous nodes and irregular users has always been a critical issue in maintaining safety, usability, and trustiness of the networks in the web environment. In general, anomalies can be categorized into three types: (1) Point Anomalies can be considered as outliers with respect to the patterns of other data, (2) Contextual Anomalies are defined as abnormal data based on their specific attributes in one context, (3) Collective Anomalies are a group of specific data instances whose simultaneous occurrence can be considered as outliers [5].

Attributed graphs can include all of the anomaly types mentioned above. Nowadays, there are different types of networks with different characteristics, requiring distinct approaches for detecting anomalies in their structures. Attributed networks entail a great variety of graphs in the real-world such as social media, and network infrastructures. Node attributes provide valuable information about characteristics of nodes in the graph, which can be very useful in various analyses. Anomaly detection on the attributed networks has been one of the trends in recent years since it involves crucial problems like fraud detection [1], and spammer detection [9].

Moreover, since labeling anomalies is a costly and roughly impossible job especially in large datasets, considering unsupervised methods for detecting anomalies is more reasonable.

Node attributes and structural features are two major sources of information that can be considered together in the methods whose goal is detecting network anomalies. Various approaches have been developed to extract structural related features of each node in a network.

This paper suggests a two-step deep learning based method for detecting anomalies which uses both structural and node attributes. This method is called Deep ${ }^{2}$ NAD (two-step deep learning method for network anomaly detection). The proposed approach utilizes node2vec [10], a particular deep learning based graph embedding technique, to extract a feature vector for each node based on its structural position in the graph in order to empower the model to detect structurally anomalous nodes.

Furthermore, Autoencoders are deep architectures that have made different unsupervised tasks feasible in machine learning. We used variational autoencoders [2], a particular probabilistic autoencoder, to learn the dominant distribution, by considering the node attributes and structural features. As anomalies are rarer cases than the normal nodes, the input data is imbalanced, so the 
VAE learns the distribution of dominant samples. In this way, the reconstruction loss for anomalies would be higher than the normal instances.

The structure of the rest of the paper is as follows: Section 2 reviews recent related works on anomaly detection in attributed networks. Section 3 is devoted to explanation of the suggested method. Experimental results are elaborated in section 4. Finally, in section 5 , we will draw the conclusion.

\section{RELATED WORKS}

Jiang et al. [12] considered both individual features and relationships between users for feeding into the Graph Convolutional Networks (GCN). By using structural features of graph and user relationships, they overcome the limitation of regular CNN models in processing non-euclidean data. The input of the model is divided into two different parts, matrix $\mathrm{F}^{\mathrm{N} \times \mathrm{D}}$ is for individual features and matrix $\mathrm{A}^{\mathrm{N} \times \mathrm{N}}$ represents the adjacency matrix. They also used a weighted function to process pure the adjacency matrix to enhance accuracy.

Chaudhary et al. [6] assumed three hypotheses for characteristics of anomalous nodes in graphs. Nodes with irregular behaviors have usually higher degree, between centrality and closeness centrality. Then at each step of evaluation, they determined thresholds for three aforementioned criteria to detect the best possible feature and threshold.

Ding et al. [7] suggested Deep Anomaly Detection on Attributed Network (DOMINANT) method, a deep autoencoder inspired by [13] that uses GCN to encode input to a latent space and then decode it to reconstruct input. They spot anomalous nodes by ranking all of them in terms of reconstruction errors after feeding to the network.

Zheng et al. [27] proposed another framework based on GCN and an attention model to consider both structure and contents of nodes for anomaly detection in dynamic graphs. They also employed a negative sampling approach to handle the lack of labeled data.

Moreover, defining an optimization problem was another way that in some research works such as [15] and [20]. For example, Peng et al. [20], tried to perform attribute selection and anomaly detection tasks by CUR matrix decomposition [18] and residual analysis.

Hu et al. [11] suggested anomaly detection method for graphs by calculating an embedding for each node. Actually, each dimension in the embedding represents a particular cluster existing in the graph.

Both supervised and unsupervised approaches were used in some works. [24] injected labeled anomalies in graph and compared two graph based methods (DNODA, CNA) and two unsupervised approaches (Isolation Forrest [17] and autoencoder [14]) performance in anomaly detection task.

An interactive approach was used in the proposed model of [8] in order to handle anomaly detection in attributed graphs. Different graph models were used in the suggested model of [4] for anomaly detection. They also used ConOut [23] and Radar [15] as their outlier detection algorithms on various graph datasets.
A new measure called normality to evaluate neighborhoods has been defined by Perozzi et al. [21]. They consider both internal consistency and external separability in calculating normality which was employed in anomaly detection in graphs.

More traditional approaches such as Local Outlier Factor (LOF) [3] can be used for detecting anomalies in graphs as well as vectorbased data. The local outlier factor can indicate how much a particular node is isolated from its neighborhood. Moreover, Xu et al. [26] designed an algorithm to detect clusters, hubs, and outliers based on the structure of the nodes in a graph.

The lack of powerful methods for extracting structural features in previous works motivates us to utilize a more complicated approach that is able to obtain information about different aspects of the graph. Moreover, the essence of the outlier detection task is dealing with a huge amount of data. Since the models proposed recently for anomaly detection used common deep learning models like vanilla autoencoders, they did not possess enough ability to gain a general distribution on a big amount of data. To overcome this issue, we aim to use VAE as a generative model. As this paper utilizes graph embedding, here, we briefly review two important network embedding methods. Achieving a comprehensive representation of the graph structure has been a matter of debate in recent years. This representation can be used for analytical procedures such as anomaly detection.

Perozzi et al. [22] devised a two-step algorithm to create embeddings for each node in a graph. First, a random walker traverses the graph to generate random walks considered as a corpus for the next process. Then, Skip-gram [19] algorithm was used to treat the random sequences as sentences and calculate the embedding for each node.

Node2vec method [10] is presented by Grover et al. for representational learning on graphs. This method can learn continuous feature representation for the nodes. In this method, there are random walks controlled by two parameters of $\mathrm{p}$ and $\mathrm{q}$ indicating the probability that use Breadth-first search (BFS) walk or Depth-first search (DFS) one. After extracting the sequence of nodes using different random walkers, all sequences are considered as sentences and input into the Skip-gram models in order to extract dense and low-dimension vectors for each node.

\section{TWO-STEP DEEP LEANING METHOD FOR NETWORK ANOMALY DETECTION}

In this study, we propose an unsupervised approach using the VAE and deep graph embedding techniques to detect anomalies in complex networks called Deep ${ }^{2} \mathrm{NAD}$. In contrast to traditional unsupervised methods such as clustering based approaches, which have a high computational cost and slow speed on a large volume of data, using VAE allows us to detect abnormal nodes in networks more efficiently. Furthermore, graph embeddings methods can extract valuable features representing a node properly. Consequently, the great success story of deep architecture motivates us to combine both approaches to detect anomalies more accurately.

\subsection{Problem Definition}

In this subsection, we formally specify the problem using the notations presented in Table 1 


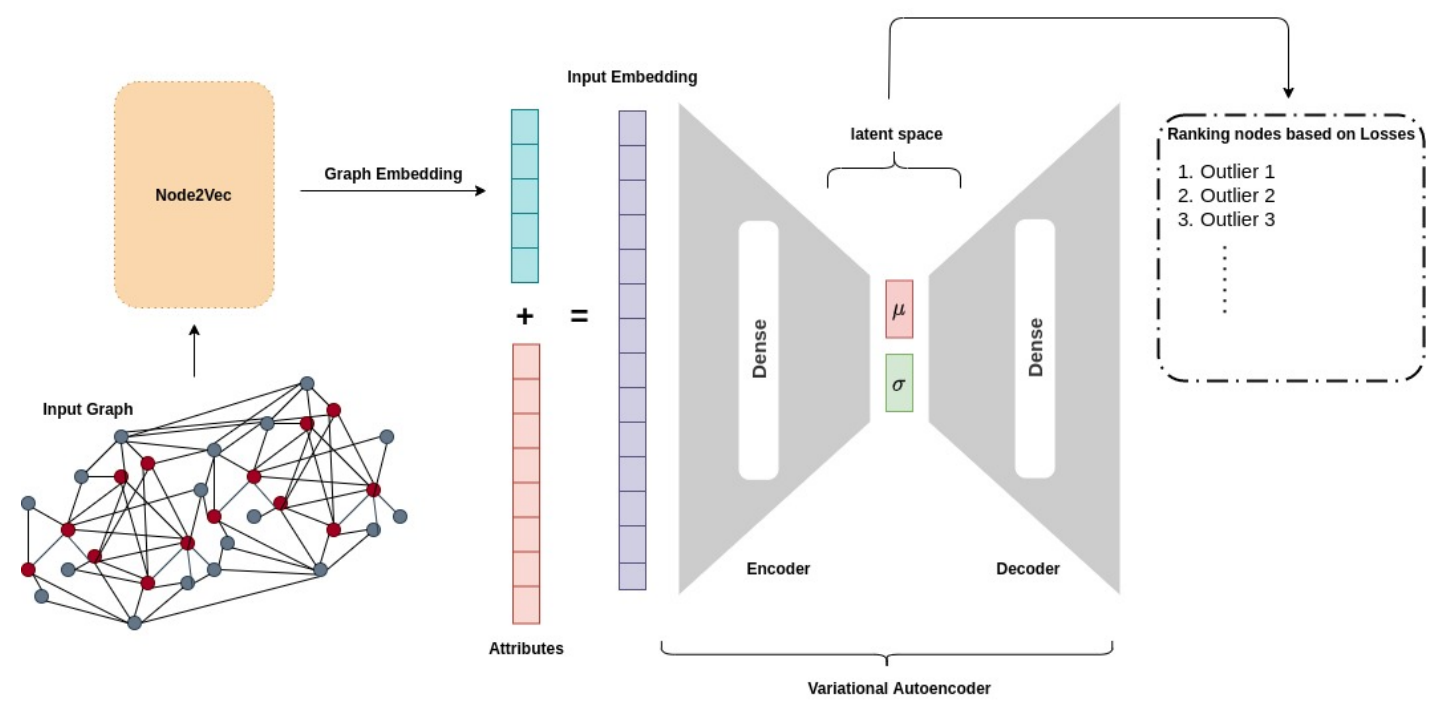

Figure 1: The architecture of our proposed method Deep ${ }^{2}$ NAD.

Table 1: The descriptions of all notation used in this paper.

\begin{tabular}{ll}
\hline Notation & Definition \\
\hline $\mathrm{G}$ & Attribute Graph \\
$\mathrm{V}$ & Set of nodes in graph \\
$\mathrm{E}$ & Set of edges in graph \\
$\mathrm{A}$ & Set of nodes' attribute vectors \\
$\mathrm{N}$ & The number of nodes \\
$d_{a}$ & The dimension of attribute vector \\
$d_{s}$ & The dimension of graph embedding \\
$\mathrm{Z}$ & Latent vector \\
\hline
\end{tabular}

Definition 1, Attribute Graph $G=\{V, E, A\}$ is defined as a unidirectional graph in which $V$ is the set of embedding vectors for nodes, $\mathrm{E}$ is the set of edges and $\mathrm{A}$ is the set of attributes by which each node is associated.

Problem: The main purpose of this paper is to detect the nodes that differ significantly in their communities based on both structure and attribute vectors. More specifically, we aim to find an optimum function $\mathrm{f}$ : Node $\rightarrow$ Score that calculates a score identifying how much the node is different from others, and a threshold $\lambda$, that nodes with grater score than $\lambda$ are anomalies.

$$
y_{i}=\left\{\begin{array}{cc}
\text { Anomaly, } & \text { if } f(\text { Node }) \geq \lambda \\
\text { Normal }, & \text { otherwise }
\end{array}\right.
$$

\subsection{Step 1: Extracting Structural Features}

Anomaly detection in graph-based data can be applied for detecting abnormal edges and nodes. In this paper, we aim to find nodes that vary from other nodes in attributes or structure. To achieve the goal, a dense representation for nodes of the graph would be required, which embeds valuable features about the structure of the node and its neighbors, so we applied the node2vec algorithm [10] on the graph to learn a representation for each node. Finally, both extracted structural features by node2vec and attribute vectors of each node are used for training a deep model employing an unsupervised approach.

\subsection{Step 2: Anomaly Detection}

After combining structural features and node attributes, we need a method for detecting anomalies. VAE is a special kind of neural network architecture that can learn particular patterns in a given dataset in an unsupervised manner. VAE is composed of two essential parts: encoder and decoder, aiming to encode the data by learning latent vectors in a lower dimensional space which leads to minimizing the loss function [2]. In our proposed method, a VAE is used for transforming input features into a low-dimensional latent representation $Z$. More specifically, the attribute matrix with the structural features builds the input vectors for our model. Considering Equation 2, inputs feed into a hidden layer. $[\mathrm{X} \mid \mathrm{A}]$ means the concatenated vectors of $\mathrm{X}$ and $\mathrm{A}$.

$$
\mathrm{H}=\operatorname{relu}\left([\mathrm{X} \mid \mathrm{A}]^{\mathrm{T}} \mathrm{W}+\mathrm{b}\right)
$$

Due to the essence of VAE that is a generative model, we put constraints on the encoder model to follow a Gaussian distribution. For this aim, two latent layers are defined to learn both mean and variance parameters of the latent distribution as follow:

$$
\begin{gathered}
\mathrm{Z}_{\text {mean }}=\mathrm{H}^{\mathrm{T}} \mathrm{W}_{\mathrm{zm}}+\mathrm{b}_{\mathrm{zm}} \\
\mathrm{Z}_{\text {logvar }}=\mathrm{H}^{\mathrm{T}} \mathrm{W}_{\mathrm{zvar}}+\mathrm{b}_{\mathrm{zvar}}
\end{gathered}
$$

Then, the model uses a sampling layer for creating the latent vector which is the low-dimensional representation of the input vector, and the decoder tries to reconstruct the input using this vector.

$$
Z=Z_{\text {mean }}+e^{0.5 \times Z_{\log \text { var }}} \times \varepsilon
$$

The latent vector connects into a decoder in order to reconstruct the input data including both attribute and structures embedding 
Table 2: The statistics of the two attributed networks.

\begin{tabular}{lll}
\hline Statistics & BlogCatalog & Flickr \\
\hline Number of nodes & 5,196 & 7,575 \\
Number of edges & 171,743 & 239,738 \\
Number of attributes & 8,189 & 12,047 \\
Number of anomalies & 300 & 450 \\
\hline
\end{tabular}

vectors, and calculating both reconstruction loss and KullbackLeibler divergence.

$$
\begin{gathered}
\hat{H}=Z^{T} W+b \\
{[\hat{X} \mid \hat{A}]=\hat{H}^{T} W+b}
\end{gathered}
$$

$\mathrm{KL}$ divergence is an approach for measuring the distance between two probability distributions. In Equation 8, $p$ is the goal distribution, $q$ is considered as the learned distribution, and $z$ is the random variable.

$$
K L[p][q]=\sum_{z}(q(z) \log (p(z) / q(z)))
$$

By using this approach, our objective function is evidence lower bound (ELBO), and our model aims to lower this value during the training process in order to learn the distribution of the input data.

$$
\begin{gathered}
\text { loss }_{\text {Reconstruction }}=\sum([X \mid A]-[\hat{X} \mid \hat{A}])^{2} \\
\text { loss } K L=-0.5 \times \sum\left(1+z_{\text {logvar }}-z_{\text {mean }}{ }^{2}-e^{z_{\text {logvar }}}\right) \\
\text { Loss }=\text { loss }_{\text {Reconstruction }}+\text { loss }_{K L}
\end{gathered}
$$

The architecture of the suggested approach is shown in Figure 1. Pure adjacency matrix is fed to the Node2vec algorithm to reach graph embeddings. The concatenation of graph embeddings and attributes is considered as input for the VAE. At the last part, nodes are sorted based on their reconstruction loss in order to detect most anomalous items in network.

\section{EXPERIMENTS}

1 This section is devoted to the evaluation of the proposed method Deep ${ }^{2}$ NAD.

\subsection{Datasets}

For the evaluation of our method, we use the same datasets as used by Ding et al. [7] (see Table 2).

In recent years, various researches has been conducted on two real-world datasets, BlogCatalog and Flickr, for evaluating proposed methods. In this paper, we evaluate the effectiveness of our models based on its performance on these two datasets. BlogCatalog is a website for sharing blogs, and users can follow each other, and form a graph. Likewise, Flickr is an image hosting service, and users by following each other can create communities (see [7] for more details).

Due to the uncertainty of conceptual similarity between datasets' labels and the concept of anomalies in graphs, we need to inject both structural and attribute anomalies using the anomaly injection method proposed by [7]. To generate structural anomalies, they connect $\mathrm{n}$ random nodes in the graph to each other for $\mathrm{m}$ times. Also, for injecting anomalies based on nodes' attributes, $\mathrm{m} \times \mathrm{n}$ nodes are selected as candidates, and for each candidate, $\mathrm{k}$ nodes are chosen from the network. For each i in the candidates set, the attribute vector of node $i$ is equal to the attribute vector of node $j$ among the $\mathrm{k}$ nodes that maximize the Euclidean Distance between $\mathrm{i}$ and $\mathrm{j}$ attribute vectors.

\subsection{Baselines}

Deep ${ }^{2}$ NAD has been evaluated by comparing its performance with some of the recent methods:

- LOF: Local outlier factor [3] is an outlier (anomaly) detection method. It is possible to use this method for detecting node outliers in a graph structure, by considering their neighborhood's characteristics.

- SCAN: This method suggested for clustering, detecting hubs and anomalies by considering the structural characteristics of nodes [26].

- Radar: The approach suggested by Li et al. [15] that handled the anomaly detection task by residual analysis.

- Amen: The method proposed by Perozzi and Akoglu [21] which is a method based on a new measure called normality for detecting outliers in graph.

- Anomalous: The method presented by Peng et al. [20] utilizes CUR decomposition to select the best instances and attributes in order to used them in anomaly detection.

- Dominant: An approach proposed by Ding et al. [7] in which a GCN-based architecture was used for processing both structural features and attributes.

\subsection{Settings and Evaluation Metrics}

The networkx package was used for processing the graph structure. Gensim and StellarGraph (https://github.com/stellargraph/ stellargraph) were employed for generating random walks and running the Word2vec algorithm to build the whole node2vec architecture. We used PyTorch library for implementing deep autoencoder VAE. Regarding what latent dimension we used in the mode, we chose the dimension of the dense layer 512 between the input and latent layer in the encoder and latent layer and output in the decoder. We set the fixed value for the dimension of latent space: 256 . Dimension for graph embedding was set at 256. Learning rate was fixed at $1 \mathrm{e}-3$ during the training.

Now, the evaluation metrics that we used for comparing numerically our model with other works should be elaborated. Three evaluation metrics AUC (area under the curve), Precision@k, and Recall@k that have been widely used in other related works for the evaluation of the models created for anomaly detection were used in our approach likewise. 
Table 3: Comparison the performance of different anomaly detection approaches using precision@K and recall@K.

\begin{tabular}{|c|c|c|c|c|c|c|c|c|}
\hline \multicolumn{9}{|c|}{ Precision@K } \\
\hline \multirow[b]{2}{*}{ K } & \multicolumn{4}{|c|}{ BlogCatalog } & \multicolumn{4}{|c|}{ Flickr } \\
\hline & 50 & 100 & 200 & 300 & 50 & 100 & 200 & 300 \\
\hline LOF & 0.300 & 0.220 & 0.180 & 0.183 & 0.420 & 0.380 & 0.270 & 0.237 \\
\hline Radar & 0.666 & 0.670 & 0.550 & 0.416 & 0.740 & 0.700 & 0.635 & 0.503 \\
\hline Anomalous & 0.640 & 0.650 & 0.515 & 0.417 & 0.790 & 0.710 & 0.650 & 0.510 \\
\hline Dominant & 0.760 & 0.710 & 0.590 & 0.470 & 0.770 & 0.730 & 0.685 & 0.593 \\
\hline Deep2NAD & 0.600 & 0.640 & 0.525 & 0.410 & 0.760 & 0.780 & 0.665 & 0.583 \\
\hline \multicolumn{9}{|c|}{ Recall@K } \\
\hline & \multicolumn{4}{|c|}{ BlogCatalog } & \multicolumn{4}{|c|}{ Flickr } \\
\hline K & 50 & 100 & 200 & 300 & 50 & 100 & 200 & 300 \\
\hline LOF & 0.050 & 0.073 & 0.120 & 0.183 & 0.047 & 0.084 & 0.120 & 0.158 \\
\hline Radar & 0.110 & 0.223 & 0.367 & 0.416 & 0.082 & 0.156 & 0.282 & 0.336 \\
\hline Anomalous & 0.107 & 0.217 & 0.343 & 0.417 & 0.087 & 0.158 & 0.289 & 0.340 \\
\hline Dominant & 0.127 & 0.237 & 0.393 & 0.470 & 0.084 & 0.162 & 0.304 & 0.396 \\
\hline Deep2NAD & 0.100 & 0.213 & 0.350 & 0.410 & 0.084 & 0.173 & 0.295 & 0.388 \\
\hline
\end{tabular}

- AUC: AUC is the area under the receiver operating characteristic curve which is plotted by two metrics True Positive Rate (TPR) and False Positive Rate (FPR). In the ROC curve, the horizontal axis is FPR and TPR is the vertical axis [16].

- Precission@k: This measure considers how much the model is accurate in the first $\mathrm{k}$ nodes returned as anomalies [25]:

Precision@k $=\frac{\text { true positives in } \mathrm{k} \text { first nodes returned as anomalies }}{\mathrm{k}}$

- Recall@k: This metric calculates the sensitivity of the model in the first $\mathrm{k}$ nodes returned as anomalies [25]:

Recall@ $\mathrm{k}=\frac{\text { true positives in } \mathrm{k} \text { first nodes returned as anomalies }}{\text { total number of anomalies }}$

\subsection{Results}

Here the proposed method is compared with other methods such as LOF, SCAN, AMEN, Anomalous, and Dominant. Table 3 illustrates the performance of our model and baselines based on Precision@k and Recall@k for k = 50, 100, 200, 300 as evaluation metrics in comparison to other methods. Additionally, Table 4 demonstrates the AUC of each model. The results of other methods are reported from [7]. As there is a trade-off between precision and recall it is better to compare different methods based on the AUC.

The results showed that Deep ${ }^{2}$ NAD method outperformed the state-of-the-art result on the Flickr dataset with AUC score of 75.21. In the BlogCatalog dataset, although the AUC of Deep ${ }^{2} \mathrm{NAD}$ is not better than that of the Dominant method, it is similar to that. It is important to note that the Dominant method uses an important parameter called $\alpha$ in the loss function that balances the impacts of the structure reconstruction error and attribute reconstruction error. This parameter is very important to set correctly, if it set very well then it can reach good results. However, finding this parameter is time-consuming and needs to try and error. We compare
Table 4: Comparison the performance of different anomaly detection approaches using AUC.

\begin{tabular}{lll}
\hline \multirow{2}{*}{ Method } & \multicolumn{2}{c}{ Dataset } \\
\cline { 2 - 3 } & BlogCatalog & Flickr \\
\hline LOF & 49.15 & 48.81 \\
SCAN & 27.27 & 26.86 \\
AMEN & 53.37 & 60.47 \\
Radar & 71.04 & 72.86 \\
Anomalous & 72.81 & 71.59 \\
Dominant & 78.13 & 74.90 \\
Deep2NAD & 72.76 & 75.21 \\
\hline
\end{tabular}

our method with the best possible value of $\alpha$ that they reported. However, Deep ${ }^{2}$ NAD model does not need such a parameter and without such a trial and error can reach this AUC in average which is better in one benchmark and comparable in the other one.

\subsection{Discussion}

The results show that the simultaneous combination of network structural information and node attribute information can help to find abnormal nodes. Of course, the extent to which this combination of information can be helpful depends entirely on the reasons why a node in the network is anomalous. A node in a network may be only structurally abnormal. For example, a person may generate an account on a social network with very common features, but then make very unusual connections with other users (and even with other anomalous users). In this case, detecting this anomaly can be possible only by considering the structural features and without considering the node attributes. Likewise, anomalous nodes in a social network may be anomalous only in terms of individual characteristics and their type of relationship with others in the network may not be structurally anomalous. In this case, there is no need to consider structural information to diagnose anomalies. But if the 
network contains both types of anomalies (similar to many real cases), then using Deep ${ }^{2}$ NAD allows us to detect mixture anomalies and is highly efficient in these conditions.

\section{CONCLUSION}

In this paper, a two-step deep method proposed for detecting anomalous nodes in attributed networks called Deep ${ }^{2}$ NAD. This method uses node2vec and variational autoencoders. At first, node2vec is utilized for extracting features for each node from the graph structure and then these features as well as the node attributes are fed into the VAE. The nodes with high reconstruction errors consider as anomalies.

Flickr and BlogCatalog datasets are two attributed networks were used evaluation of Deep ${ }^{2}$ NAD. We compared our strategy with other state-of-the-art models done for anomaly detection in attributed networks. The results show that Deep ${ }^{2}$ NAD can effectively find the anomalous nodes in node attributed networks.

For future work, we suggest considering an explainable deep learning method that can indicate the reason for being an anomaly. For example, it is interesting to know which one is important in being an anomaly: the structure of the network or the node attributes.

\section{REFERENCES}

[1] Akoglu, L., Tong, H., Koutra, D.: Graph based anomaly detection and description: a survey. Data mining and knowledge discovery 29(3), 626-688 (2015)

[2] An, J., Cho, S.: Variational autoencoder based anomaly detection using reconstruction probability. Special Lecture on IE 2(1), 1-18 (2015)

[3] Breunig, M.M., Kriegel, H.P., Ng, R.T., Sander, J.: Lof: identifying density-based local outliers. In: Proceedings of the 2000 ACM SIGMOD international conference on Management of data. pp. 93-104 (2000)

[4] Campos, G.O., Moreira, E., Meira Jr, W., Zimek, A.: Outlier detection in graphs: A study on the impact of multiple graph models. Computer Science and Information Systems 16(2), 565-595 (2019)

[5] Chandola, V., Banerjee, A., Kumar, V.: Anomaly detection: A survey. ACM computing surveys (CSUR) 41(3), 1-58 (2009)

[6] Chaudhary, A., Mittal, H., Arora, A.: Anomaly detection using graph neural networks. In: 2019 International Conference on Machine Learning, Big Data, Cloud and Parallel Computing (COMITCon). pp. 346-350. IEEE (2019)

[7] Ding, K., Li, J., Bhanushali, R., Liu, H.: Deep anomaly detection on attributed networks. In: Proceedings of the 2019 SIAM International Conference on Data Mining. pp. 594-602. SIAM (2019)

[8] Ding, K., Li, J., Liu, H.: Interactive anomaly detection on attributed networks. In: Proceedings of the Twelfth ACM International Conference on Web Search and
Data Mining. pp. 357-365 (2019)

[9] Fakhraei, S., Foulds, J., Shashanka, M., Getoor, L.: Collective spammer detection in evolving multi-relational social networks. In: Proceedings of the 21th acm sigkdd international conference on knowledge discovery and data mining. pp. 1769-1778 (2015)

[10] Grover, A., Leskovec, J.: node2vec: Scalable feature learning for networks. In: Proceedings of the 22nd ACM SIGKDD international conference on Knowledge discovery and data mining. pp. 855-864 (2016)

[11] Hu, R., Aggarwal, C.C., Ma, S., Huai, J.: An embedding approach to anomaly detection. In: 2016 IEEE 32nd International Conference on Data Engineering (ICDE). pp. 385-396. IEEE (2016)

[12] Jiang, J., Chen, J., Gu, T., Choo, K.K.R., Liu, C., Yu, M., Huang, W., Mohapatra, P.: Anomaly detection with graph convolutional networks for insider threat and fraud detection. In: MILCOM 2019-2019 IEEE Military Communications Conference (MILCOM). pp. 109-114. IEEE (2019)

[13] Kipf, T.N., Welling, M.: Semi-supervised classification with graph convolutional networks. arXiv preprint arXiv:1609.02907 (2016)

[14] Kramer, M.A.: Nonlinear principal component analysis using autoassociative neural networks. AIChE journal 37(2), 233-243 (1991)

[15] Li, J., Dani, H., Hu, X., Liu, H.: Radar: Residual analysis for anomaly detection in attributed networks. In: IJCAI. pp. 2152-2158 (2017)

[16] Ling, C.X., Huang, J., Zhang, H., et al.: Auc: a statistically consistent and more discriminating measure than accuracy. In: Ijcai. vol. 3, pp. 519-524 (2003)

[17] Liu, F.T., Ting, K.M., Zhou, Z.H.: Isolation forest. In: 2008 Eighth IEEE International Conference on Data Mining. pp. 413-422. IEEE (2008)

[18] Mahoney, M.W., Drineas, P.: Cur matrix decompositions for improved data analysis. Proceedings of the National Academy of Sciences 106(3), 697-702 (2009)

[19] Mikolov, T., Sutskever, I., Chen, K., Corrado, G.S., Dean, J.: Distributed representations of words and phrases and their compositionality. In: Advances in neural information processing systems. pp. 3111-3119 (2013)

[20] Peng, Z., Luo, M., Li, J., Liu, H., Zheng, Q.: Anomalous: A joint modeling approach for anomaly detection on attributed networks. In: IJCAI. pp. 3513-3519 (2018)

[21] Perozzi, B., Akoglu, L.: Scalable anomaly ranking of attributed neighborhoods. In: Proceedings of the 2016 SIAM International Conference on Data Mining. pp. 207-215. SIAM (2016)

[22] Perozzi, B., Al-Rfou, R., Skiena, S.: Deepwalk: Online learning of social representations. In: Proceedings of the 20th ACM SIGKDD international conference on Knowledge discovery and data mining. pp. 701-710 (2014)

[23] Sánchez, P.I., Müller, E., Irmler, O., Böhm, K.: Local context selection for outlier ranking in graphs with multiple numeric node attributes. In: Proceedings of the 26th International Conference on Scientific and Statistical Database Management. pp. 1-12 (2014)

[24] Vengertsev, D., Thakkar, H.: Anomaly detection in graph: Unsupervised learning, graph-based features and deep architecture (2015)

[25] Vuong, B.Q., Lim, E.P., Sun, A., Le, M.T., Lauw, H.W., Chang, K.: On ranking controversies in wikipedia: models and evaluation. In: Proceedings of the 2008 International Conference on Web Search and Data Mining. pp. 171-182 (2008)

[26] Xu, X., Yuruk, N., Feng, Z., Schweiger, T.A.: Scan: a structural clustering algorithm for networks. In: Proceedings of the 13th ACM SIGKDD international conference on Knowledge discovery and data mining. pp. 824-833 (2007)

[27] Zheng, L., Li, Z., Li, J., Li, Z., Gao, J.: Addgraph: Anomaly detection in dynamic graph using attention-based temporal gcn. In: IJCAI. pp. 4419-4425 (2019 